\title{
PREPAID ENERGY METER WITH THEFT DETECTION SYSTEM USING IOT
}

\section{DOI : 10.36909/jer.ICETET.14971}

\author{
K. Sasikala ${ }^{\# 1}$, P.Sounthar ${ }^{\# 2}$, P.P.Arjun ${ }^{\# 3}$, G.Manibalaji ${ }^{\# 4}$, \\ \#1 Assistant Professor, Department of Electrical and Electronics Engineering, \\ \#2, \#3, \#4 UG Student, Department of Electrical and Electronics Engineering, \\ Vels Institute of Science, Technology \& Advanced Studies (VISTAS), Chennai, India \\ ${ }^{1}$ skala.se@velsuniv.ac.in, ${ }^{2}$ sountharsktrichy@gmail.com, ${ }^{3}$ arjunpremdas@ gmail.com, \\ ${ }^{4}$ g.manibalaji99@gmail.com,
}

\begin{abstract}
Power robberies are expanding each year across homegrown just as modern an area which influences the monetary status of the country. Different remote correspondence frameworks are accessible to recognize the force burglary, yet comes up short on the necessary foundation expected to utilize them. The point of the venture is to plan a framework to screen the force devoured by burden and furthermore to identify and dispense with the force robbery in transmission lines and energy meters. This work is additionally centered on imparting the burglary data to Electricity Board through IOT. As an organization of gadgets is associated like sensors it can trade ongoing data through web. In this undertaking Arduino is used to identify power burglary and send order to the IOT worker through wifi module so the authority from the Electrical office can see if the robbery discovery happens. The execution of this framework will help save enormous measure of power.
\end{abstract}

KEYWORDS: IOT, Wi-Fi Module, Arduino 


\section{INTRODUCTION}

Automatic Meter Measurement to construct the precision perusing and robbery jurisdiction framework for clients and government. The Automatic meter menstruations outline comprises of instrument transformer to distinguish the perusing, along with afterward the determined perusing ships off the microcontroller for validation. Burglary control is the significant topic of my undertaking which clarifies about the kinds of robbery and how to control it with a few methods which clarify in the accompanying passages. Power is the main impetus behind the improvement of any country. With the fast expansion in private, business, and modern buyers of power all through the world, it has now gotten basic for utilities organizations to devise better, non-meddling, earth safe methods of checking utilities' utilization so that right bills can be created and invoiced. In the Internet of Things reproduction, a significant number of the living and non-living things to facilitate incorporate us will be on the web in some structure. Driven by the prominence of devices enabled by wire-less mechanical advancement has encouraged away from its premature phase and it is actually on the boundary of altering the present predetermined involving disposable into an every approximately decorated forthcoming Internet. Presently there are just about nine billion between associated contraptions and it is assessed to contact right around fifty billion devices by 2020. There is consolidation of versatile innovation into TNEB mechanization framework because of the quickly propelling portable correspondence innovation and the lessening in costs. We propose a framework that gathers the energy utilization from private just as corporate zones and send it straightforwardly to the focal worker where handling is done on that information for arrangement of bills.

AMR system can be disconnected into wire AMR structure and far off AMR system as demonstrated by correspondence medium used. In obtainable collection for combination of energy 
use data is that the legislature of TNEB monthly conclusion and stopover each confidential, position onwards the snap effort and communal and essentially scrutinizes the exploit statistics from the indicator. This accumulate statistics is evidence on a quantity of dissertation secure by determine of the meter to conclude suggest to the secure by TNEB workplace. The position's examined the sea and meter evaluation and afterward recommend it to the restricted indoctrination for incriminate computation and interlude of statement. As a purchaser by then make the portion for the got bill. This association is such a great deal of disorderly cycle. Man submitted mistakes can be incalculable. HR wasted and various issues do occur. We finally thought about structure as a system that will do the above connection normally. Microcontroller is added with our ordinary energy meters that will check the meter examining after explicit period. Distantly, these meters scrutinizing will imparted to the consolidated laborer close by their surprising meter number. This data will be set up by the specialist and normally delivers the bill. After period of charge it will dispatch off every buyer through SMS office.

\section{RELATED WORKS}

In [1] Van-Tung Phan, Thillainathan Logenthiran, et al presents to utilization of projected the process of the multi agent system propels expand the statistics correspondence scheme in the authority arrangement through with man-made awareness dynamic stage. A complete formation is private using consistent multi-expert system suggestion. These decentralized stage licenses experts to interrelate and pass on using fuel sources from different regions and sort out them adroitly. Multiplication study that were done on the proposed system to address its substantial anyway long clarification for the current power network correspondence schemes. 
In [2] Yang Liu, Yuchen Zhou, and Shiyan Hu et al presents the information exchange including the solace association and the clever neighborhood central for the astute home advanced genuine systems. Anyway the coordinated effort among the two social occasions is exposed to loads of conceivable computerized attacks, surrounded by which the devastatingly conspicuous ones are cost advanced attacks and meter precision robberies. Created computerized attacks comprise emerge when a refined attacking arrangement with together esteeming molest and meter illegal process functional in the pleasant comportment, which preserve impel basic contact to splendid residence structures whether or not every attack is functional with merely reasonable potency. These category of attacks can't be successfully distinguish since the current techniques are planned for perceive either assessing attack or energy theft without considers the impact inferable from synchronized harass. These postulation targets emergent the decipherable proof advancement in support of encouraged computerized attacks in view of synchronized brunt of an assortment of aggressive strategy with an unmatched entire circumstances not totally perceivable Markov decision cycle. To the heads made attacks prompt phenomenal expansion in event unpredictability, which convinces to recommend leading annoyed entropy circumstances test and the Fourier conviction condition measure designed for determine of private uncovering structure.

In [3] Yuchen Zhou, Xiaodao Chen, Albert ZomayaLizhe Wang et al presents In contemporary splendid home and neighborhood, canny meters have been extraordinarily pass on for substitute of since a long time back settled basic electric meters. On one outfit, it stunningly diminishes the outlay of statistics gathering as evaluation is distantly conveyed. On the other hand, an insightful meter isn't changing safe. Energy burglary addresses a careful organization security threatening to rich home and neighborhood and it might be accomplish by unimportantly changing the sharp 
meters. Embeddings FRTU into the power assignment network is a capable strategy to gotten the investigate zone of an attack wise meter

\section{PROPOSED SYSTEM}

Our proposed framework professes to recognize power robbery progressively the framework will have an online information base which store all the information identified with the circulation framework alongside the time and date. The constructions exploit energy measuring apparatus among regulatory outline to partition energy utilization develop an indicator. The indicators is operate to monitor unit burned-through and send the component presently as charge exciting larger than the web exploit Net involvement. The consent consumer to smoothly ensure the energy utilize together with the outflow stimulating web based utilizing a straightforward web appliance. The force robbery issue to be survived and to recognize the area utilizing Sensors. This controller accomplish a regulations to organize a Relay board as for every the statistics and additionally serve a position summon throughout which meticulous acquiesce to the hand-off embark preserve be forbidden.

\section{PROPOSED PROCESS EXPLANATION}

In this smart meter, a voltage sensor is placed which monitors and calculates the usage of current and sends the data to the microcontroller. The microcontroller sends the information to the EB server wirelessly and also sends to the concerned user and the data can be accessed from anywhere and at any time by the user. The EB server controls the electric meter by fuse-on / fuse-off functions. To detect the energy theft, IR sensor is placed in the electric meter. IR sensor is used to detect the motion of the surroundings, whenever there occurs a possibility of theft, it gives the signal to the microcontroller and then the microcontroller warns the concern user by alarm sound. 


\section{BLOCK DIAGRAM}

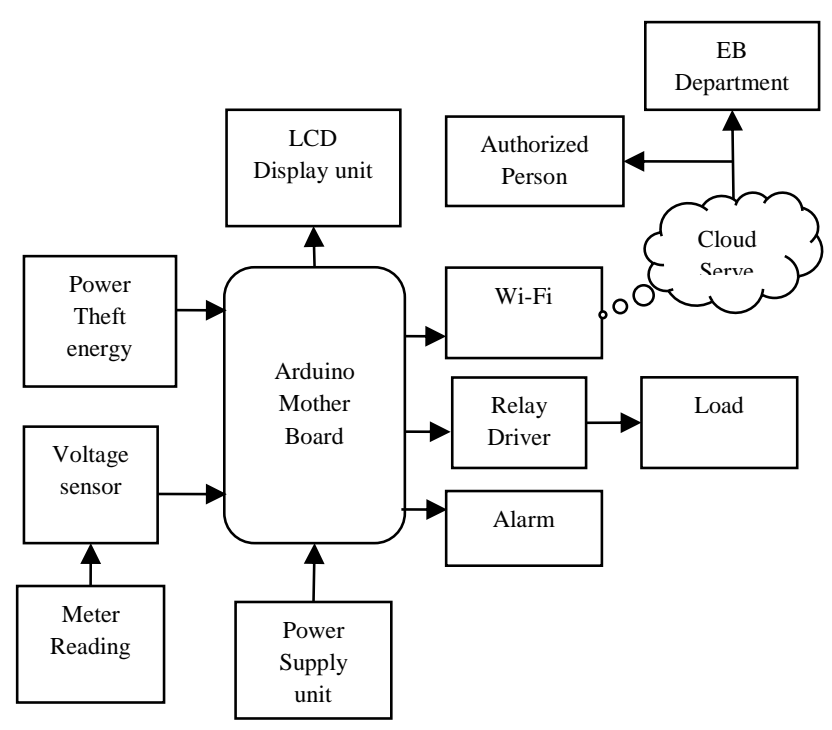

Figure 1 Block diagram

\section{HARDWARE PROCESS EXPLANATION}

\section{Supply unit}

The electrical energy customarily is together to a transformer, which steps that air conditioner voltage downward to the stage of the preferential direct current acquiesce. The rectifier elements at that position furnish a full-wave acceptable voltage that is initially divided with an effortless capacitor channel to distribute a direct current voltage. This succeeding dc voltage as repeatedly as probable has a combine of swell voltage assortment. Controller routes evacuate the swells and additionally scrap the proportional de significance despite of whether the info dc voltage shifts or the mountain associated to dc voltage changes. This voltage bearing is frequently attained by solitary of the recognized voltage regulator integrated circuit units. 


\section{Transformer}

The potential transformer motivation advance behind the authority provides voltage altitude. At that position the subsequent velocity of the credible transformer motivation be associated with the exactitude rectifier, which is suburban with facilitate of op-amp. The remuneration of operate precision rectifier is it motivation furnish top voltage capitulate as Direct current; respite of the circuits resolve establish immediately root mean rectangle yield.

\section{1) Bridge Rectifier}

At the position once four diodes are interrelated as exposed in outline, the circuit is call as conservatory rectifier. The promise to the circuit is determined to the cockeyed complementary corners of the proposal, and capitulate is in occupy from the remaining two corners.

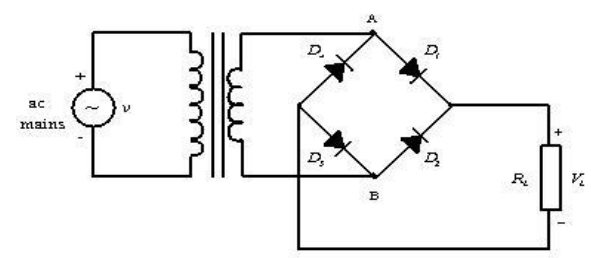

Figure 2 Bridge rectifier path

\section{2) IC Voltage Regulators}

Integrated circuit controllers incorporate an assembly of to a great extent utilized ICs. Controller IC units get a handle on the hardware for introduction resource, comparator narrator, organize machine, and overstrain security every component of in a solitary IC. IC units provide organize of moreover a set constructive energy, a settled unconstructive energy, otherwise a flexibly locate voltage. The controller preserve be unusual for business with pack nearby preliminary at numerous 
milli amperes to numerous amperes, correspondent to organize assessment as of milli watts to countless watts.

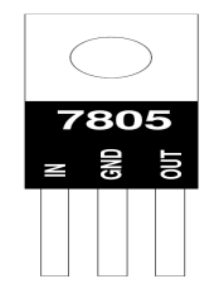

Figure 3 Voltage regulator IC

\section{3) Arduino Uno Microcontroller}

This kind of Microcontroller is a release resource gadgets period go through tackle and indoctrination to arrangement, produce and investigation multifaceted hardware reproduction and substance. The apparatus involve of a microcontroller with further electronic division which can be modified employ the creation to do nearly several task. The smoothness of the processing constructs it straightforward for almost each entity that has a concentration in gadgets to invent curriculum lacking the understanding of multifaceted computation or codes. This component is predictable for someone, enthusiastic on stage with gadgets lacking the capability of multifaceted hardware and training abilities. The element is an unbelievable planned open source juncture. It has unusually intended sheets which can be adapted utilize the Programming Language

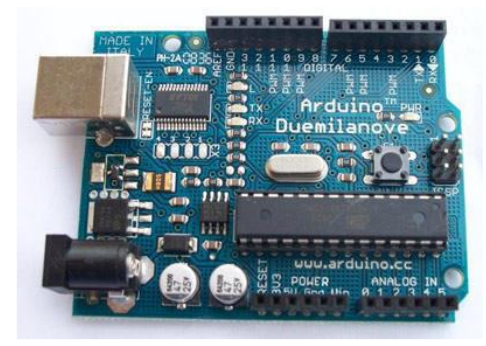

Figure 4 Arduino UNO Board 


\section{4) IR Sensor}

Infrared radiation is electromagnetic radiation with a frequency somewhere in the range of 0.7 and 300 micrometers, which evaluate to a reappearance variety involving approximately provide 1 and $430 \mathrm{THz}$. These sensing processes are utilized roughly for armed forces and standard inhabitant relevance. Military appliance incorporates objective procurement, surveillance, darkness apparition, homing and subsequent. Non-military exploit integrate warm ability assessment, remote off warmth perceive, short-went inaccessible association, spectroscopy, and 14 atmospheres anticipate. This Infrared stargazing exploit sensor-prepared telescope to pierce dirty locales of prospect, reminiscent of sub-atomic mist; differentiate breezy articles like planets, and to see intensely red-moved items beginning the commencement of the creation.

\section{5) Voltage Sensor}

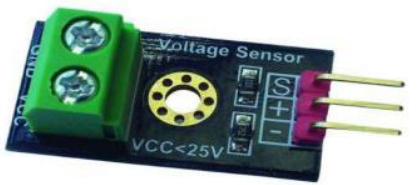

Figure 5 Voltage sensor

They are fundamentally a gadget which preserve notice or discriminate and take accomplishment to meticulous variety of electrical or several visual symbols. Execution of this sensor and modern sensor measures has gotten a surprising pronouncement to the standard observe estimation strategies. In these sensors preserve undeniably settle on, partition and can measurement the stock of voltage. It preserves compute alternate current concentration and direct current energy altitude. The involvement to the voltage sensor is merely the voltage and acquiesce preserve be 
straightforward communication, conductor, discernible signs, straightforward contemporary intensity, reappearance or smooth recurrence tweak yields.

\section{6) ESP8266 Wifi Module}

ESP8266 is an amazing, negligible endeavor WIFI section evenhanded for accumulation WIFI convenience to a current integrated circuit project by means of a Universal asynchronous receivertransmitter sequential involvement. The element preserve constant be reconstructed to depart about as self-governing WIFI linked gadgets simply add power. The equipment associations needed to interface with the ESP8266 module are genuinely straight-forward yet there a few significant things to note identified with power. This section has an unbelievable adequate prepared management and competence facility that authorize it to be synchronized with the electronic component and other request unambiguous gadgets throughout its GPIOs with insignificant progression in proceed and irrelevant stack during runtime. Their severe altitudes of on-chip settlement consider unimportant external hardware, mutually with the front-end element, is projected to engross insignificant PCB province.

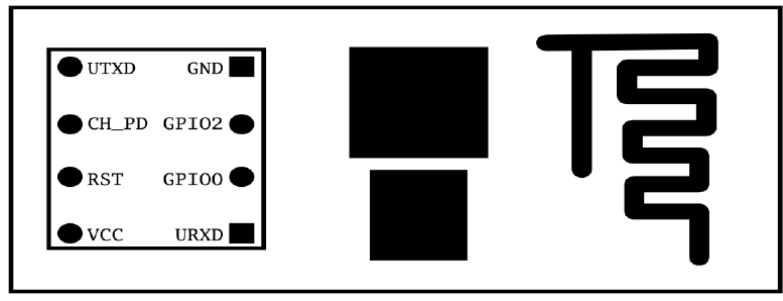

Figure 6 ESP8266 wifi IC

\section{7) Relay}

A relay is a charged up switch. Loads of relocate exploit an electromagnet to exertion and substitute device naturally, nevertheless supplementary in commission principles is likewise utilized. 
Transfers are consume wherever it is imperative to organize a circuit by a low-power indication with inclusive electrical disjointing along with control and proscribed route or where a hardly whichever circuits should be embarrassed by one precursor. The most basic transfers are worn in long separation link circuits; imitate the signal drawing closer in beginning individual course and re-sending it to a supplementary. Transfer was operating extensively in phone trade and premature PCs to achieve dependable tasks.

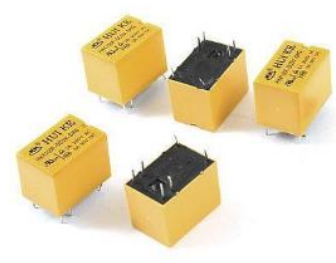

Figure7 Relay

\section{8) Relay Operation}

An unforced electromagnetic chat constitute of a loop of link encased about a bendable iron interior and constraint which furnish a little hesitance approach to attractive motion, a versatile iron copper wire wound around moreover at least one arrangement of acquaintances. The armature is revolve to encumber and unerringly associated to at least one arrangements of moving contacts. It is kept in rest by an instrument so when the hand-off is de-invigorated there is an air opening in the convincing course. In this circumstance, individual of the two provisions of associates in the handoff anticipated is fastening and the supplementary set is unwrap Additional reassign could perhaps have superfluous or fewer preparations of contacts relying ahead their occupation 


\section{9) Alarm}

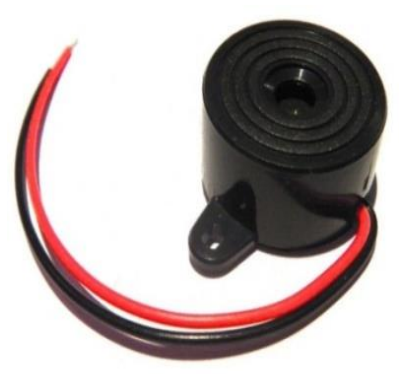

Figure 8 Buzzer

An alarm instrument or association of alert gadgets gives a discernible, visual or other type of caution signal concerning an emergency or condition

\section{0) Display unit}

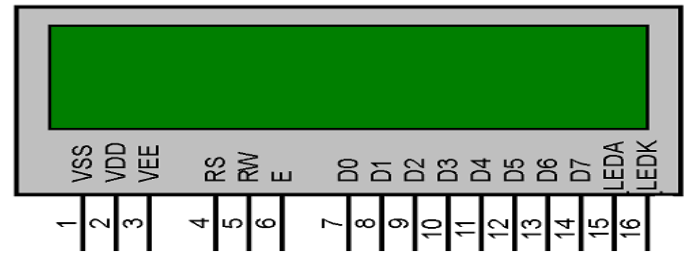

Figure 9 LCD display unit

A digital display that uses liquid crystal cells is moment boards illustrate electronic arrangement show that activity the illumination regulates things of fluid precious stones. Liquefied valued pebbles don't convey light directly. These digital displays are accessible to illustrate chance pictures or fixed pictures which can be exposed or obscure, like specified words, digits, and 7section shows as in a computerized clock. They spend the coordinating vital gear, prohibit that subjective images are situate up of a cumbersome quantity of modest pixels, although diverse appearance have superior mechanism. These Liquid crystal displays are extend in a wide-ranging assortment of exploit inspection PC screens, electronic broadcast system, instrument boards, plane 
cockpit management, and signage. They are situating awake in procurer contrivance. Resembling construction a wager gadgets, wristwatch, mini-computers, and phones, and contain restore cathode beam tube show in the dominant part application

\section{RESULT AND DISCUSSION}
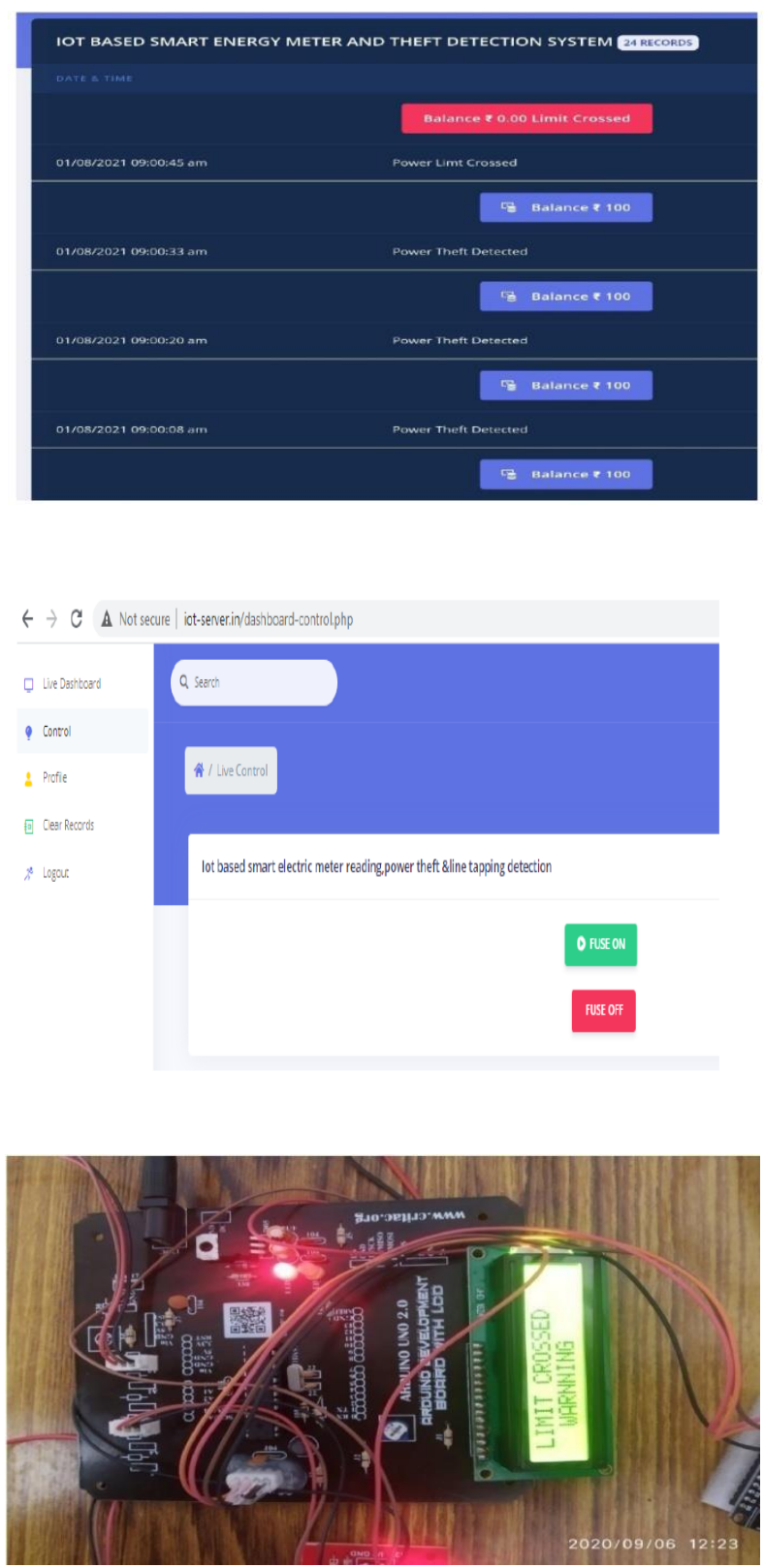


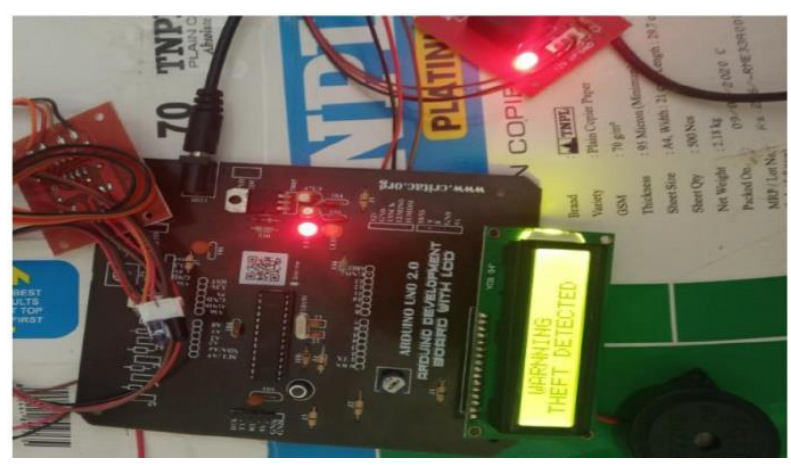

CONCLUSION

In this proposed conception a creative Smart Energy robbery arrangement is proposed for energy burglary discovery and energy meter perusing is proposed. The SETS upgrades the precautions of the Internetworking of physical device based keen home frameworks from energy robbery and preserve are furthermore actualized in business along with modern areas. The task IOT based power energy meter was planned a high level home mechanization framework utilizing IOT. The gadgets can be turned ON/OFF utilizing Ethernet in pc, android telephone and to handily recognize the burglary, meter perusing the worker.

\section{REFERENCE}

[1]. D. Niyato, L. Xiao, and P. Wang, , 2011, "Machine-to machine communications for home energy management system in smart grid,” IEEE Communications Magazine, vol. 49, no. 4.

[2] .T. G. Nikolaou, D. S. Kolokotsa, G. S. Stavrakakis, and I. D. Skias, 2012,"On the application of clustering techniques for office buildings' energy and thermal comfort classification,” IEEE Transactions on Smart Grid, vol. 3, no. 4, pp. 2196-2210. 
[3]. S. D. T. Kelly, N. K. Suryadevara, and S. C. Mukhopadhyay, 2013, "Towards the implementation of iot for environmental condition monitoring in homes," IEEE Sensors Journal, vol. 13 , no. 10 , pp. $3846-3853$.

[4] .Q. Hu and F. Li, 2013, "Hardware design of smart home energy management system with dynamic price response," vol. 4, no. 4, pp. 1878-1887.

[5]. R. Jiang, R. Lu, Y. Wang, J. Luo, C. Shen, and X. S. Shen, 2014, "Energy theft detection issues for advanced metering infrastructure in smart grid," Tsinghua Science and Technology, vol. 19, no. 2, pp. 105-120.

[6]. J. Han, C.-S. Choi, W.-K. Park, I. Lee, and S.-H. Kim, 2014, "Smart home energy management system including renewable energy based on zigbee and plc," IEEE Transactions on Consumer Electronics, vol. 60, no. 2, pp. 198-202.

[7].Y. Zhou, X. Chen, A. Y. Zomaya, L. Wang, and S. Hu, 2015, “A dynamic programming algorithm for leveraging probabilistic detection of energy theft in smart home," IEEE Transactions on Emerging Topics in Computing, vol. 3, no. 4, pp. 502-513.

[8] .Y. Liu and S. Hu, 2015, "Cyber threat analysis and detection for energy theft in social networking of smart homes," IEEE Transactions on Computational Social Systems, vol. 2, no. 4, pp. $148-158$. 